\title{
Arteritis Infective, CTCAE
}

National Cancer Institute

\section{Source}

National Cancer Institute. Arteritis Infective, CT CAE. NCI Thesaurus. Code C143297.

A disorder characterized by an infectious process involving an artery. 